\title{
Primed for a response
}

Dendritic cell (DC) vaccines autologous DCs pulsed with tumour antigens ex vivo and re-injected into patients - have been used in an attempt to induce immune responses in patients with cancer. However, DC vaccines have so far shown limited efficacy in tumour immunotherapy, and the factors that might contribute to their efficacy are not well understood. Mitchell and Batich et al. have found that priming the vaccine site with a recall antigen improves the efficacy of DC vaccines in patients with glioblastoma.

The authors conducted a randomized and blinded trial in which 12 patients with glioblastoma were randomly assigned to receive vaccine site pre-conditioning with either tetanus/diphtheria (Td) toxoid

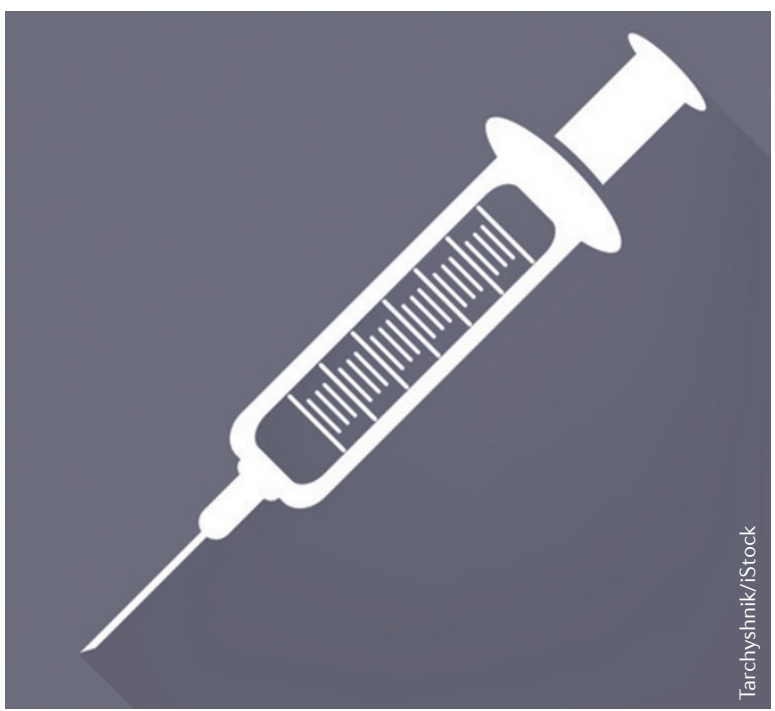

(a potent recall antigen) or mature DCs prior to vaccination with DCs pulsed with Cytomegalovirus phosphoprotein 65 (pp65) RNA, which is expressed in $>90 \%$ of glioblastomas but not in normal brain. Patients who received Td had significantly more pp65-specific DCs in vaccine-site draining lymph nodes (VDLNs) than patients primed with mature DCs, which is important because DCs must migrate to lymph nodes in order to activate $\mathrm{T}$ cell-driven immune responses. Patients who received Td also had significantly increased progression-free and overall survival; half of the Td cohort ( 3 of 6 patients) did not progress and were still alive $>36$ months after randomization. Furthermore, migration of DCs to the VDLNs correlated with improved progression-free and overall survival.

To analyse the mechanism of this response, the authors examined mice pre-conditioned with $\mathrm{Td}$ that then received DCs pulsed with ovalbumin (OVA) RNA. These mice also had increased DCs in VDLNs compared with control, Td-naive, mice. Depletion of various immune cell subsets indicated that $\mathrm{CD} 4^{+} \mathrm{T}$ cells were crucial for DC migration, and DC migration could be enhanced in Td-naive mice by administration of Td-activated $\mathrm{CD} 4^{+} \mathrm{T}$ cells. The authors examined serum levels of several chemokines and found that levels of chemokine (C-C motif) ligand 3 (CCL3) were increased in both patients and mice in response to
Td treatment and reduced in mice if $\mathrm{CD}^{+} \mathrm{T}$ cells were depleted. Migration of DCs to VDLNs following Td preconditioning was significantly reduced in $\mathrm{Ccl3}^{-/-}$mice compared with wildtype mice; this could not be rescued by administration of Td-activated $\mathrm{CD}^{+}$ T cells, and exogenous CCL3 administration could only induce DC migration following Td pre-conditioning, indicating that both CCL3 and $\mathrm{CD} 4^{+}$ $\mathrm{T}$ cells are required.

To examine possible therapeutic responses in a cancer model system, the authors injected mice subcutaneously with OVA-expressing B16 melanoma cells. Td pre-conditioning and administration of OVA-specific DCs significantly delayed tumour growth compared with mice that were not pre-conditioned with $\mathrm{Td}$, or mice pre-conditioned with $\mathrm{Td}$ and given DCs pulsed with a control antigen. Tumour growth was not affected in $\mathrm{Ccl3}^{-/-}$mice, confirming the importance of this chemokine.

These data suggest that $\mathrm{DC}$ vaccine responses in patients with glioblastoma might be improved through the use of Td pre-conditioning and further suggest that migration of DCs to VDLNs might serve as a biomarker to predict patient response.

Sarah Seton-Rogers, Chief Editor, Nature Reviews Cancer This article is modified from the original in Nature Rev. Cancer (http://dx.doi.org/10.1038/nrc3945)

ORIGINAL RESEARCH PAPER Mitchell, D. A. \& Batich, K. A. et al. Tetanus toxoid and CCL3 improve dendritic cell vaccines in mice and glioblastoma patients. Nature 519, 366-369 (2015) 